\title{
Transoral robotic surgery (TORS): a new tool for high risk tracheostomy decannulation
}

\author{
La chirurgia robotica transorale (TORS): una nuova applicazione \\ nelle decannulazioni ad alto rischio \\ F. MONTEVECCHI ${ }^{1}$, G. CAMMAROTO², G. MECCARIELLO ${ }^{1}$, P.T. HOFF³ , R.M. CORSO ${ }^{4}$, C. GALLETTI ${ }^{5}$, \\ M.F.H. AL-RAWASHDEH ${ }^{1}$, C. VICINI \\ ${ }^{1}$ Head and Neck Department, ENT \& Oral Surgery Unit, G.B. Morgagni, L. Pierantoni Hospital, Forlì, Infermi \\ Hospital, Faenza, ASL of Romagna, Italy; ${ }^{2}$ Department of Otorhinolaryngology, University of Messina, Italy; \\ ${ }^{3}$ Department of Otolaryngology, Head and Neck Surgery, University of Michigan, Ann Arbor, Michigan, USA; \\ ${ }^{4}$ Emergency Department, Anaesthesia and Intensive Care Section, "GB Morgagni-L. Pierantoni" Hospital, Forlì, \\ Italy; ${ }^{5}$ Department of Anaesthesiology, University of Messina, Italy
}

\section{SUMMARY}

Tracheostomy decannulation has always been considered a procedure with an attendant risk, especially in patients with a reduced upper airway diameter as is commonly observed in the obstructive sleep apnoea (OSA) population. We report on 4 cases where transoral robotic surgery (TORS) helped in the management of long-term cannulated patients. The aims of our paper are: 1. To demonstrate how the otolaryngology team can help identify patients at high risk for decannulation failure; and 2. To demonstrate how TORS may aid in the decannulation process of patients at high risk for failure due to severe tongue base hypertrophy. From our experience, TORS appears to offer an effective option to aid in the decannulation of patients with a severe hypertrophy of the base of tongue and floppy epiglottis.

KEY WORDS: Transoral Robotic Surgery • Tongue base $\bullet$ Tracheostomy

\section{RIASSUNTO}

La decannulazione è sempre stata considerata una procedura con un certo grado di rischio, specie nei pazienti con ridotti diametri delle via aeree, come nel caso della sindrome delle apnee ostruttive (OSA). Presentiamo 4 casi nei quali la chirurgia robotica transorale (TORS) ha permesso un appropriato management di pazienti tracheotomizzati da divers mesi. Gli obiettivi del nostro lavoro sono: 1. Dimostrare come il team otorinolaringoiatrico possa favorire il riconoscimento di pazienti ad alto rischio di decannulazione inefficace e 2 . Evidenziare il ruolo nella TORS nel trattamento dell'ipertrofia della base della lingua, responsabile dell'ostruzione delle vie aeree superiori. Dalla nostra esperienza la TORS appare una tecnica efficace nella decannulazione di pazienti affetti da ipertrofia della base della lingua e da epiglottide flottante.

PAROLE CHIAVE: Chirurgia robotica transorale $\bullet$ Base della lingua $\bullet$ Tracheostomia

Acta Otorhinolaryngol Ital 2017;37:46-50

\section{Introduction}

Tracheostomy decannulation has always been considered a procedure with an attendant risk, especially in patients with a reduced upper airway diameter as is commonly observed in the obstructive sleep apnoea (OSA) population. From a recent survey, most clinicians would consider decannulation failure, if reinsertion of an artificial airway occurs within 48 to 96 hours following planned tracheostomy removal ${ }^{1}$.

The cause for re-intubation or a new tracheostomy intervention after decannulation is multifactorial: level of consciousness, cough effectiveness, secretions and oxygenation seem to be the most important factors contributing to the success or failure. In addition, anaesthesiol- ogists consider the difficulty of intubation to be another important contributing element ${ }^{1}$. Cormack and Lehane, in particular, described anatomic conditions associated with difficult intubation (grades 3 and 4) ${ }^{2}$. The main anatomic obstacles are a narrow incisor distance, micrognathia, and hypertrophy of the base of tongue (BOT) with or without floppy epiglottis. The latter, in particular, is very common in patients affected by OSA. In our Institutions (Forlì, Italy and Ann Arbor, Michigan, USA) more than 650 cases have been treated with trans-oral robotic surgery (TORS).

The Morgagni-Pierantoni Hospital is a tertiary care hospital located in Forli, Italy. A multidisciplinary airway board has been developed to assist in the management of 
tracheostomy patients and others with long-term airway requirements. The board includes otolaryngologist and anaesthesiologists of our Institution and takes advantage of consulting from others Italian and international centres. As part of this effort, the board evaluates known difficult airway patients and patients for whom difficulty in tracheostomy decannulation is anticipated or who have failed prior attempts at decannulation. This team approach has led to the application of TORS and other techniques in providing patients with effective airway management leading to successful decannulation.

Recently, this technique has gained popularity as an effective therapeutic option both for OSA and head and neck cancer ${ }^{3-5}$. The aims of our paper are: 1 . To demonstrate how the otolaryngology team can help identify patients at high risk for decannulation failure and 2. To demonstrate how TORS may aid in the decannulation process of patients at high risk for failure due to severe tongue base hypertrophy. We present the following case series.

\section{Case series}

We report on 4 cases where TORS helped in the management of long-term cannulated patients.

All patients gave their consent to the procedure. TORS was carried out by the same team (C.V. and F.M.) with an Intuitive da Vinci robot. The operative setting was the same as that described by Weinstein ${ }^{5}$ for the tongue base neoplasms. The robot is set up on the right side of the patient. The eyes and teeth are protected by means of specific devices. After the insertion of a mouth gag, the da Vinci robotic arms are placed in the oral cavity. Visualisation is achieved with a 30x magnification, 3-dimensional endoscope. Surgery begins with the visualisation of the epiglottis to orientate the surgeon. Then a piecemeal resection of the BOT is performed using a step-by-step approach. First the medial and paramedial portions of the tongue base are addressed and then the lateral parts. In this way, it is possible to identify and preserve the noble structures.

Case \#1: D.S.F., male, 69 years, came to our attention in October 2014 for evaluation of tracheostomy removal. The tracheostomy was performed in January 2014 for a severe respiratory impairment that occurred after a stroke. Several unsuccessful trials of decannulation were attempted before our evaluation.

The patient's past medical history was complicated type 2 diabetes, arterial hypertension, ischaemic cardiopathy (he underwent 7 coronary stents in 1995) and both alcohol and tobacco addiction.

The endoscopic examination highlighted hypertrophy of the BOT and oedema of the epiglottis causing near complete occlusion of the upper airway (Fig. 1). As part of his evaluation for failed decannulation, the patient also underwent both a CT scan and an MRI scan of the neck with contrast (Fig. 2).

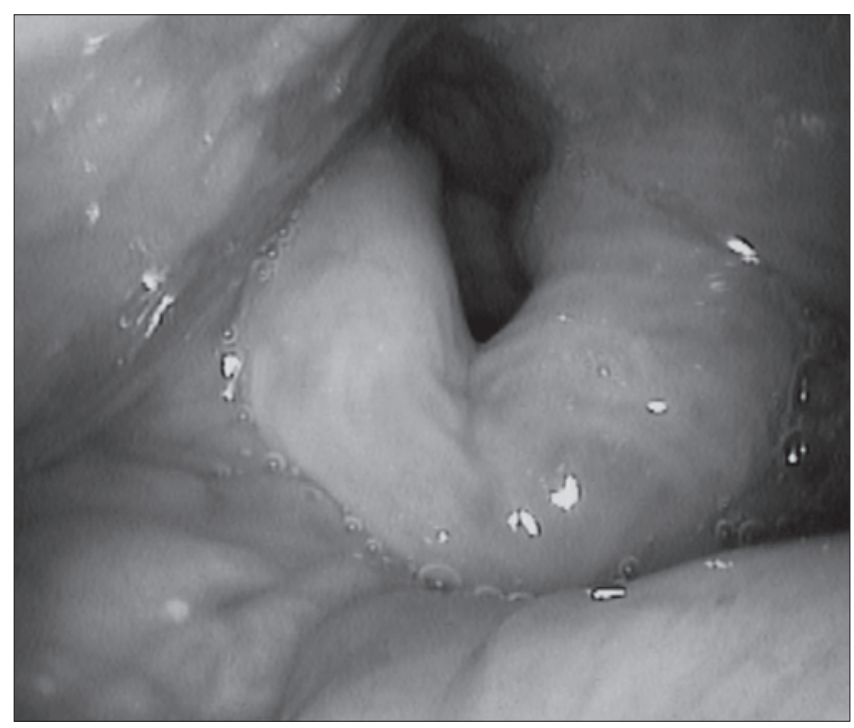

Fig. 1. Endoscopic examination before TORS highlighted hypertrophy of the BOT associated to an oedema of the epiglottis occluding almost completely the upper airway.

The case was discussed at the multidisciplinary airway conference where it was recommended that the patient undergo TORS BOT reduction and supraglottoplasty (SGP). The patient underwent TORS BOT resection and SGP in October 2014; a total of $12 \mathrm{mls}$ of lingual tissue was resected. The patient was successfully decannulated in January 2015. At 6 months follow-up, the patient did not complain any further symptoms and did not require replacement of the tracheostomy (Fig. 3).

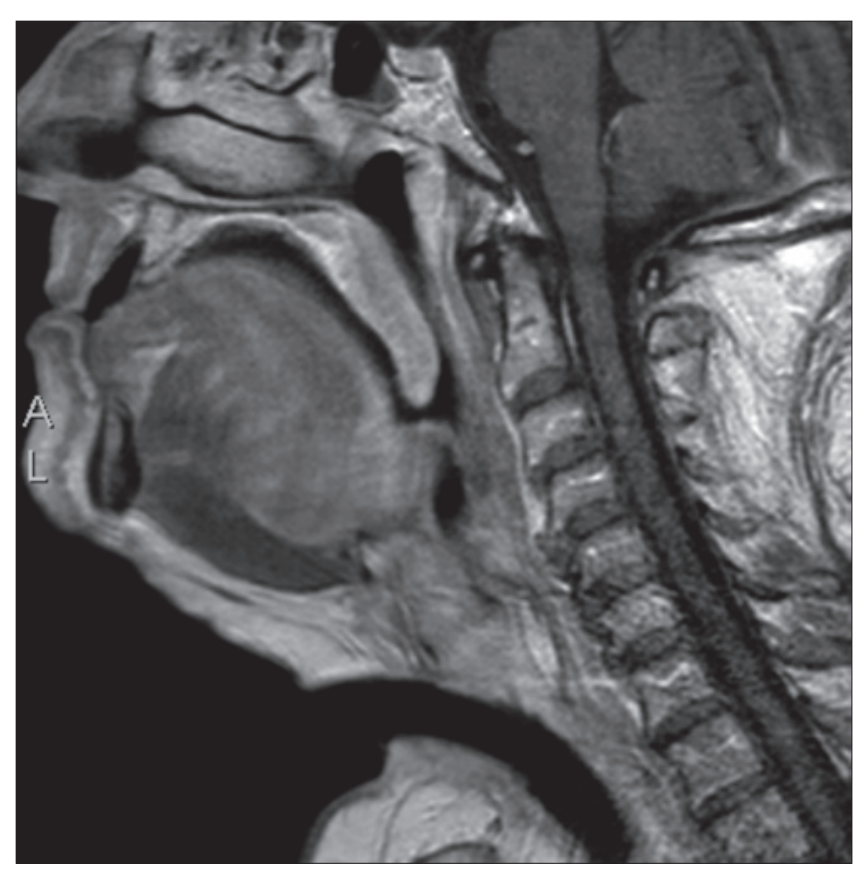

Fig. 2. MRI examination highlighted a muscular verticalised BOT with a significant narrowing of the upper airway. 


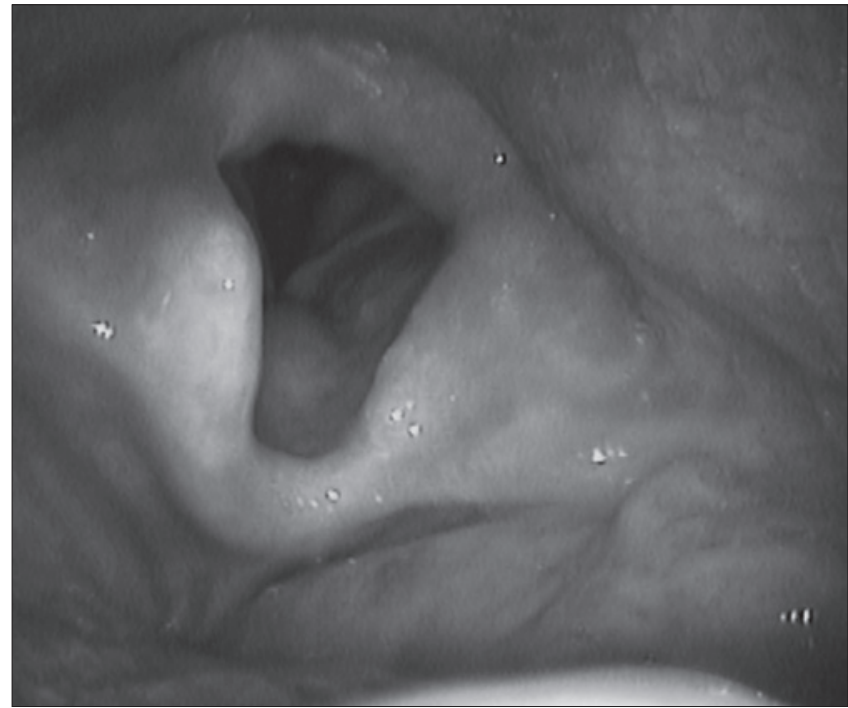

Fig. 3. Endoscopic view after 6 months of follow-up.

Case \#2: M.MA. is a 61-year-old woman who developed a severe post-operative oedema of the BOT in January 2014 following microlaryngoscopy and biopsy of BOT lymphatic tissue. For this reason, the patient underwent an urgent tracheostomy performed by otolaryngologists at another Institution.

Several months later, the patient was referred for consultation. Interestingly, the patient had also undergone a previous cervical spine surgery secondary to severe trauma 10 years before the above-reported event. The patient's physical examination demonstrated hypertrophy of the BOT (Fig. 4).

Considering the patient's limited cervical mobility, reduced upper airway volumes due to BOT hypertrophy, several unsuccessful trials of tracheostomy removal, and

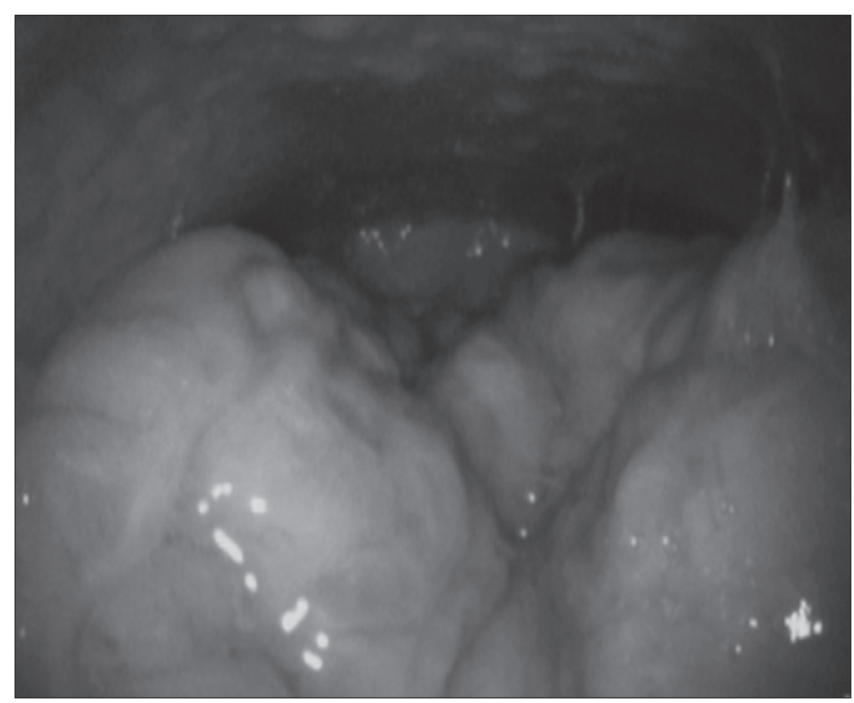

Fig. 4. Endoscopic view shows a lymphatic hypertrophy of the BOT before TORS. the high risk of re-intubation failure, our team opted for TORS. In September 2014 the patient underwent TORS BOT resection and SGP; a total volume of $7 \mathrm{mls}$ of lymphoid tissue was removed from the BOT. Three months later in December 2014 the patient was successfully decannulated, and six months later, upon routine followup, she continues to do well without a tracheostomy (Fig. 5).

Case \#3: S.V. is a 43-year-old man affected by achondroplasia. As part of his anatomic phenotype he presented with a pronounced hypoplasia of the upper maxilla. He underwent a tracheostomy due to acute respiratory failure likely associated with his progressive, severe pattern of OSA. The Apnoea Hypopnoea Index (AHI) was 84, Oxygen Desaturation Index (ODI) 87, Lowest Oxygen Saturation (LOS) 60\%, Epworth Scale Score (ESS) 12 and Body Mass Index (BMI) 25.

The patient came to our attention for the evaluation of his OSA syndrome and the possibility of tracheostomy removal. The endoscopic examination highlighted hypertrophy of the BOT completely obstructing the hypopharynx.

Our team recommended a comprehensive evaluation of the upper airway using drug induced sedation endoscopy (DISE) to precisely identify the sites of obstruction contributing to both his OSA and potential decannulation failure $^{6}$. Using the NOHL classification system described by Vicini et al., the patient was found to have severe (75\%) transverse (lateral) collapse at the level of the oropharynx (O) and complete (100\%) circumferential collapse at the hypopharynx $(\mathrm{H})^{7}$.

The patient underwent TORS BOT resection in October 2015 to treat the OSA syndrome and to allow a safer decannulation procedure. A total of $5 \mathrm{mls}$ of tissue was robotically removed from the BOT.

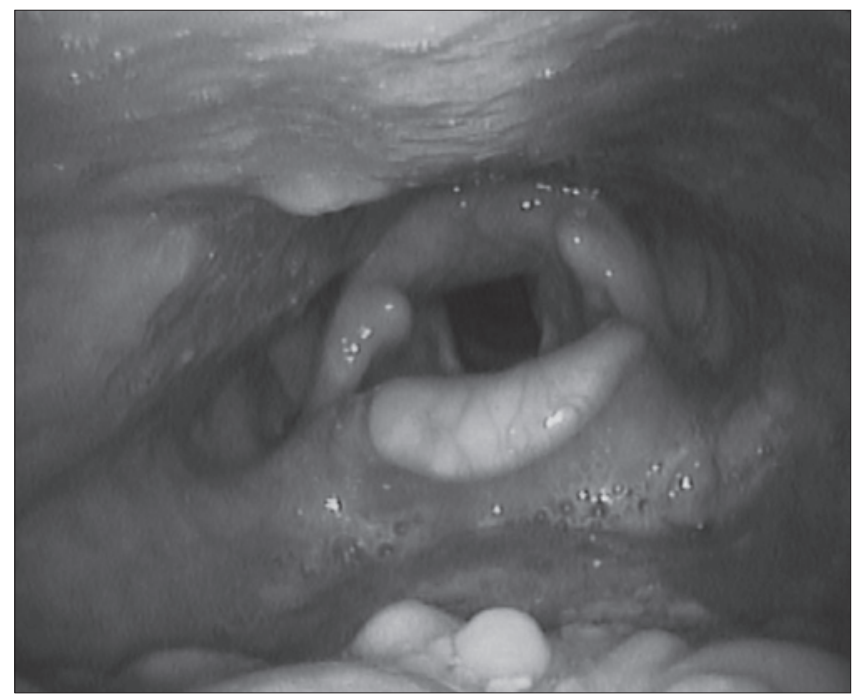

Fig. 5. Endoscopic view after 6 months of follow-up. 
In December 2015, the tracheostomy was removed and the patient underwent repeat polysomnography; a significant improvement of the sleep parameters were registered: AHI 16.5, ODI 17.8, LOS 88\%. At 6 months follow-up, the patient did not require replacement of the tracheostomy and reported subjective resolution of OSA symptoms. Case \#4: T.L. is a 49-year-old male who underwent a tracheostomy for prolonged intubation (1 week) due to respiratory failure following an epileptic crisis. As part of his evaluation for decannulation, endoscopic evaluation revealed a $40 \%$ subglottic stenosis, probably due to the tracheostomy, significant hypertrophy of the BOT and a floppy epiglottis.

In September 2015 the patient underwent a TORS BOT resection with the removal of $18 \mathrm{mls}$ of lymphoid tissue and a SGP to stabilise the epiglottis. In November 2015, the patient underwent serial laser excisions of the suprastomal tissue, and was successfully decannulated. At 6 months follow-up, the patient did not complain of further symptoms and no relapses were highlighted.

\section{Discussion}

TORS has been shown to be an effective and safe procedure for the treatment of OSA in patients with hypertrophy of the BOT and floppy epiglottis ${ }^{34}$. A low rate of major complications, such as intra-operative or post-operative massive bleeding, has been reported ${ }^{34}$. On the other hand, $14.2 \%$ of patients do report transient hypogeusia ${ }^{3}$. Furthermore, much attention has focused on TORS as a surgical option for head and neck cancer, in particular, for oropharyngeal and laryngeal carcinomas, reporting promising outcomes ${ }^{5}$.

In this paper, a new role for TORS in the management of long-term tracheostomy patients is reported. The indications for placement of a tracheostomy tube are several and include prolonged mechanical ventilation, impaired neurologic status, excessive secretions, and severe upper-airway obstructions ${ }^{8}$. Tracheostomy decannulation is generally accepted when there is no longer a need for airway protection or mechanical ventilation ${ }^{9}$. Specific decannulation strategies are frequently institution-dependent. Some authors consider it once the patient has had the tracheostomy tube plugged for 48 hours or more, whereas others consider it once a speaking valve is tolerated ${ }^{10}$.

Endoscopic inspection of the airway prior to decannulation, although not essential, can be helpful. In one study, $67 \%$ of patients with tracheostomies were found to have airway abnormalities during bronchoscopy (i.e. tracheal granulomas, tracheomalacia, tracheal stenosis and vocal cord dysfunction) ${ }^{11}$.

The epiglottis and tongue base may present additional challenges in successful decannulation. In some cases, anatomical variants preclude easy intubation. Cormack and Lehane, describe anatomic conditions where severe difficulty during intubation can be anticipated (grades 3 and 4). Several anaesthesiologists consider the difficulty of intubation an influencing element in the evaluation of decannulation.

Patients at risk for reintubation following decannulation should be carefully evaluated so that this risk is minimised. A multidisciplinary approach including otolaryngologists and anaesthesiologists must play a lead role. The role of bedside endoscopy, imaging and DISE should be considered on an individualised basis. In our series, three patients came to our attention several months after a prolonged tracheostomy due to difficult reintubation.

The most common airway abnormality in our series was significant hypertrophy of the BOT and a floppy epiglottis. In our opinion, this clinical pattern may be an obstacle for future intubations and revision tracheostomy for repositioning of the endotracheal tube, which carries a higher risk of complications (i.e. tracheomalacia), could be required.

In the second patient, hypertrophy of the BOT was accompanied by reduced cervical mobility, while significant hypoplasia of the upper maxilla characterised the third case, and subglottic stenosis the fourth patient.

All these anatomic patterns may represent an obstacle to effective decannulation and/or to any attempt of re-intubation. In fact, the first two patients experienced several unsuccessful trials of tracheostomy removal before undergoing TORS. The third patient was also affected by OSA and refused the use of positive airway pressure therapy before undergoing tracheostomy. In this case, TORS was strongly indicated and led to two satisfying results: an amelioration of the apnoea and a safer and effective decannulation.

\section{Conclusions}

In conclusion, from our experience TORS appears to offer an effective option to aid in the decannulation of patients with severe hypertrophy of the BOT and floppy epiglottis. In our opinion, physicians should be aware of the potential pathological role of these two anatomic conditions and the possible use of TORS as a safe and effective tool for the treatment of these patients.

\section{References}

1 Stelfox HT, Crimi C, Berra L, et al. Determinants of tracheostomy decannulation: an international survey. Critical Care 2008;12:R26.

2 Cormack RS, Lehane J. Difficult tracheal intubation in obstetrics. Anaesthesia 1984;39:1105-11.

3 Vicini C, Montevecchi F, Campanini A, et al. Clinical outcomes and complications associated with TORS for OSAHS: a benchmark for evaluating an emerging surgical technology in a targeted application for benign disease. ORL J Otorhinolaryngol Relat Spec 2014;76:63-9. 
4 Hoff PT, D'Agostino MA, Thaler ER. Transoral robotic surgery in benign diseases including obstructive sleep apnea: Safety and feasibility. Laryngoscope 2015;125:1249-53.

5 Weinstein GS, O’Malley BW Jr, Magnuson JS, et al. Transoral robotic surgery: a multicenter study to assess feasibility, safety, and surgical margins. Laryngoscope 2012;122:1701-7.

6 De Vito A, Carrasco M, Vanni A, et al. European position paper on drug-induced sedation endoscopy (DISE). Sleep Breath 2014;18:453-65.

7 Vicini C, De Vito A, Benazzo M, et al. The nose oropharynx hypopharynx and larynx (NOHL) classification: a new system of diagnostic standardized examination for OSAHS patients. Eur Arch Otorhinolaryngol 2012;269:1297-300.
8 Kollef MH, Ahrens TS, Shannon W. Clinical predictors and outcomes for patients requiring tracheostomy in the intensive care unit. Crit Care Med 1999;27:1714-20.

9 Dunn PF, Goulet RL. Endotracheal tubes and airway appliances. Int Anesthesiol Clin 2000;38:65-94.

10 St John RE, Malen JF. Contemporary issues in adult tracheostomy management. Crit Care Nurs Clin North Am 2004;16:413-30.

11 Law JH, Barnhart K, Rowlett W, et al. Increased frequency of obstructive airway abnormalities with longterm tracheostomy. Chest 1993;104:136-8.

Received: March 5, 2016 - Accepted: April 6, 2016

Address for correspondence: Giovanni Cammaroto, Department of Otorhinolaryngology, University of Messina, via Consolare Valeria 1, 98100, Messina, Italy. Tel./Fax +39090 2212251. E-mail: giovanni.cammaroto@ hotmail.com 